\title{
COMPACTIFICATION, AND BEYOND, OF COMPOSITION OPERATORS ON HARDY SPACES BY WEIGHTS
}

\author{
Pascal Lefèvre, Daniel Li, Hervé Queffélec and Luis Rodríguez-Piazza
}

\author{
Université d'Artois, Laboratoire de Mathématiques de Lens (LML) UR 2462 \\ \& Fédération Mathématique des Hauts-de-France FR 2037 CNRS, Faculté Jean Perrin \\ Rue Jean Souvraz, S.P. 18, F-62 300 Lens, France; pascal.lefevre@univ-artois.fr \\ Université d'Artois, Laboratoire de Mathématiques de Lens (LML) UR 2462 \\ \& Fédération Mathématique des Hauts-de-France FR 2037 CNRS, Faculté Jean Perrin \\ Rue Jean Souvraz, S.P. 18, F-62 300 Lens, France; daniel.li@univ-artois.fr \\ Université Lille Nord de France, USTL, Laboratoire Paul Painlevé U.M.R. CNRS 8524 \\ \& Fédération Mathématique des Hauts-de-France FR 2037 CNRS \\ F-59655 Villeneuve-d'Ascq Cedex, France; Herve.Queffelec@univ-lille.fr
}

Universidad de Sevilla, Facultad de Matemáticas, Departamento de Análisis Matemático \& IMUS

Calle Tarfia s/n 41012 Sevilla, Spain; piazza@us.es

\begin{abstract}
We study when multiplication by a weight can turn a non-compact composition operator on $H^{2}$ into a compact operator, and when it can be in Schatten classes. The $q$-summing case in $H^{p}$ is considered. We also study when this multiplication can turn a compact composition operator into a non-compact one.
\end{abstract}

\section{Introduction}

Let $\varphi: \mathbf{D} \rightarrow \mathbf{D}$ be an analytic self-map and $C_{\varphi}: H^{2} \rightarrow H^{2}$ be the associated composition operator $f \mapsto f \circ \varphi$. For $w \in H^{2}$, the multiplication operator $M_{w}$ is defined formally by $f \mapsto w f$ and the weighted composition operator by $f \mapsto w(f \circ \varphi)$. It is known (see [5] for instance) that twisting $C_{\varphi}$ by some $M_{w}$ can improve its compactness properties, and even its membership in Schatten classes $S_{p}$ or the decay of its approximation numbers [7, Theorem 2.3].

In this note, we study, in a rather qualitative way, the following problem: given a symbol $\varphi$, when can we find a non-trivial $w \in H^{2}$ such that $M_{w}$ has a smoothing effect on $C_{\varphi}$, namely when is $M_{w} C_{\varphi}$ compact if $C_{\varphi}$ was not? Or the other way round: when can we find $w$ such that $M_{w} C_{\varphi}$ is not compact if $C_{\varphi}$ was?

In [13, Proposition 2.4], it is proved that for $M_{w} C_{\varphi}$ to be compact for some $w \in H^{2}(w \neq \equiv)$, it is necessary that

$$
m\left(\left\{\left|\varphi^{*}\right|=1\right\}\right)=0,
$$

where $m$ is the normalized Lebesgue measure on $\mathbf{T}$ and $\varphi^{*}$ the boundary values function of $\varphi$. On the other hand, in order that $M_{w} C_{\varphi}$ be Hilbert-Schmidt for some $w \in H^{2}, w \neq \equiv 0$, it is sufficient that

$$
\int_{\mathbf{T}} \log \left(1-\left|\varphi^{*}\right|\right) d m>-\infty
$$

https://doi.org/10.5186/aasfm.2021.4602

2020 Mathematics Subject Classification: Primary 47B33; Secondary 46B28.

Key words: Approximation numbers, composition operator, compactification, decompactification, Hilbert-Schmidt operator, $p$-summing operators, Schatten classes. 
[13, Proposition 2.5]. Note that (1.1) means that $\varphi$ is not an exposed point of the unit ball of $H^{\infty}$ [1], and that (1.2) means that it is not an extreme point of this unit ball [4, Theorem 7.9].

There is a gap between these two conditions. The purpose of this work to fill this gap in several respects, this filling explaining in passing the initial gap.

In Section 3, we show that condition (1.1) is necessary and sufficient to have a compact weighted composition operator. We also give examples showing how small approximation numbers we can obtain. In Section 4, we show that condition (1.2) is necessary and sufficient to get a Hilbert-Schmidt weighted composition operator, and we show that it is also necessary and sufficient for getting a weighted composition operator in some, or all, Schatten classes. In Section 5, we consider the case of $H^{p}$ spaces and study the nuclearity and the summing properties of the weighted composition operators. In Section 6 we show that a composition operator can become non-compact by weighting it if and only if the image of the symbol touches the boundary of the unit disk.

\section{Notation}

Let $\mathbf{D}$ be the open unit disk. The Hardy space $H^{p}, 1 \leq p<\infty$, is the space of analytic functions $f: \mathbf{D} \rightarrow \mathbf{C}$ such that

$$
\|f\|_{p}^{p}:=\sup _{0<r<1} \frac{1}{2 \pi} \int_{0}^{2 \pi}\left|f\left(r \mathrm{e}^{i t}\right)\right|^{p} d t<\infty .
$$

Such functions have non-tangential limits $f^{*}\left(\mathrm{e}^{i t}\right)$ almost everywhere on $\mathbf{T}=\partial \mathbf{D}$ and we have

$$
\|f\|_{p}^{p}=\frac{1}{2 \pi} \int_{0}^{2 \pi}\left|f^{*}\left(\mathrm{e}^{i t}\right)\right|^{p} d t .
$$

For $p=2, H^{2}$ is equivalently the space of analytic functions in $\mathbf{D}$ that can be written $f(z)=\sum_{n=0}^{\infty} c_{n} z^{n}$ with $\|f\|_{2}^{2}=\sum_{n=0}^{\infty}\left|c_{n}\right|^{2}<\infty$. In the sequel, for convenience, we write simply $\|\cdot\|_{2}=\|\cdot\|$.

Any analytic self-map $\varphi: \mathbf{D} \rightarrow \mathbf{D}$ induces a bounded operator $C_{\varphi}: H^{p} \rightarrow H^{p}$, called the composition operator of symbol $\varphi$.

For $w \in H^{p}$, the multiplication operator $M_{w}$ is defined, formally, by $M_{w} f=w f$, and the weighted composition operator $M_{w} C_{\varphi}$ by $\left(M_{w} C_{\varphi}\right)(f)=w(f \circ \varphi)$. Note that to get $M_{w} C_{\varphi}: H^{p} \rightarrow H^{p}$, it is necessary to have $w \in H^{p}$ since $\left(M_{w} C_{\varphi}\right)(\mathbb{1})=w$. Throughout this paper it will be assumed that $w \in H^{p}$, and that $w \not \equiv 0$. This membership is not sufficient in general; however $w \in H^{\infty}$ is sufficient (but not necessary!), since $H^{\infty}$ is the set of multipliers of $H^{p}$. Note that we may consider the bounded operator $M_{w} C_{\varphi}$, even if $M_{w}$ is not bounded.

Except in Section 5, we work only with the Hilbert space $H^{2}$.

For convenience, we will adopt in this paper the following terminology.

Definition 2.1. We say that the symbol $\varphi$ is

- compactifiable if $M_{w} C_{\varphi}$ is compact for some $w \in H^{2}$ with $w \not \equiv 0$;

- decompactifiable if $M_{w} C_{\varphi}$ is bounded but not compact for some $w \in H^{2}$.

For $\xi \in \mathbf{T}=\partial \mathbf{D}$ and $0<h<1$, the Carleson window $W(\xi, h)$ is defined as

$$
W(\xi, h)=\{z \in \mathbf{D} ; 1-h \leq|z| \text { and }|\arg (z \bar{\xi})| \leq \pi h\} .
$$


If $\mu$ is a positive measure on $\overline{\mathbf{D}}$, the Carleson function of $\mu$ is

$$
\rho_{\mu}(h)=\sup _{\xi \in \mathbf{T}} \mu[\overline{W(\xi, h)}] .
$$

The measure $\mu$ is called a Carleson measure when $\rho_{\mu}(h)=\mathrm{O}(h)$, and a vanishing Carleson measure when $\rho_{\mu}(h)=\mathrm{o}(h)$. By the Carleson embedding theorem, this is equivalent to say that the canonical inclusion $J_{\mu}: H^{2} \rightarrow L^{2}(\mu)$ is respectively bounded or compact.

It is convenient to coin the Hastings-Luecking box $\widetilde{W}(\xi, h) \subseteq W(\xi, h)$ defined by

$$
\widetilde{W}(\xi, h)=\{z \in \mathbf{D} ; 1-h \leq|z|<1-h / 2 \text { and }-\pi h<\arg (z \bar{\xi}) \leq \pi h\} .
$$

We denote $m$ the Haar measure (normalized Lebesgue measure) of T. For a symbol $\varphi, m_{\varphi}=\varphi^{*}(m)$ is the pull-back measure of $m$ by $\varphi^{*}: \mathbf{T} \rightarrow \mathbf{C}$, the (almost everywhere defined) radial limit function associated with $\varphi$ :

$$
\varphi^{*}(\xi)=\lim _{r \rightarrow 1^{-}} \varphi(r \xi) .
$$

By definition $m_{\varphi}(B)=m\left[\varphi^{*-1}(B)\right]$ for all Borel sets $B \subseteq \overline{\mathbf{D}}$. This measure $m_{\varphi}$ is always a Carleson measure, due to the Littlewood subordination principle.

The Carleson function of $\varphi$ is that of $m_{\varphi}$ and is denoted $\rho_{\varphi}$ :

$$
\rho_{\varphi}(h)=\sup _{\xi \in \mathbf{T}} m\left(\varphi^{*-1}[\overline{W(\xi, h)}]\right) .
$$

When the composition operator $C_{\varphi}$ is compact on $H^{2}$, we have $\left|\varphi^{*}\right|<1$ a.e., and $m_{\varphi}$ is supported by $\mathbf{D}$. Moreover, $m_{\varphi}$ is then a vanishing Carleson measure.

Recall that a compact operator $T$ between separable Hilbert spaces $H_{1}$ and $H_{2}$ is in the Schatten class $S_{p}=S_{p}\left(H_{1}, H_{2}\right), p>0$, if $\sum_{n>1}\left[s_{n}(T)\right]^{p}<\infty$, where $\left(s_{n}(T)\right)$ is the sequence of singular numbers of $T$, i.e. the eigenvalues, arranged in non-increasing order, of $|T|=\sqrt{T^{*} T}$. For $p=2, S_{2}\left(H_{1}, H_{2}\right)$ is the Hilbert-Schmidt class.

Let us also recall that, for $p \geq 2$, we have $T \in S_{p}$ if and only if $\sum_{n}\left\|T e_{n}\right\|^{p}<\infty$ for every orthonormal basis $\left(e_{n}\right)$ of $H_{1}$, and, for $p \leq 2$, we have $T \in S_{p}$ if and only if $\sum_{n}\left\|T e_{n}\right\|^{p}<\infty$ for some orthonormal basis $\left(e_{n}\right)$ of $H_{1}$ (see [6] for instance). It follows that if $S, T: H_{1} \rightarrow H_{2}$ are two compact operators such that $\|S x\| \leq\|T x\|$ for all $x \in H_{1}$, then, for all $p>0, T \in S_{p}$ implies $S \in S_{p}$.

We recall Luecking's theorem [14].

Theorem 2.2. (Luecking's theorem) Let $\mu$ be a positive Borel measure on D. Then the canonical inclusion $J_{\mu}: H^{2} \rightarrow L^{2}(\mu)$ is in the Schatten class $S_{p}, p>0$, if and only if

$$
\sum_{n=0}^{\infty} \sum_{j=0}^{2^{n}-1}\left[2^{n} \mu\left(\widetilde{W}_{n, j}\right)\right]^{p / 2}<\infty,
$$

where $\widetilde{W}_{n, j}=\widetilde{W}\left(\mathrm{e}^{2 j i \pi / 2^{n}}, 2^{-n}\right)$.

Let us point out that the above condition can be replaced by the following variant [8, Proposition 3.3]:

$$
\sum_{n=0}^{\infty} \sum_{j=0}^{2^{n}-1}\left[2^{n} \mu\left(W_{n, j}\right)\right]^{p / 2}<\infty,
$$

where $W_{n, j}=W\left(\mathrm{e}^{2 j i \pi / 2^{n}}, 2^{-n}\right)$.

As usual, the notation $A \lesssim B$ means that $A \leq c B$ for some positive constant $c$, and $A \approx B$ means that $A \lesssim B$ and $B \lesssim A$. 


\section{Compactification}

Theorem 3.1. An analytic self-map $\varphi: \mathbf{D} \rightarrow \mathbf{D}$ is compactifiable if and only if $m\left(\left\{\left|\varphi^{*}\right|=1\right\}\right)=0$.

Proof. The necessary part is proved in [13, Proposition 2.4]. Let us recall the easy proof of this fact.

Indeed, suppose that $T=M_{w} C_{\varphi}$ is compact and that $\left|\varphi^{*}\right|=1$ on $E$, with $m(E)>0$. Since $\left(z^{n}\right)_{n}$ converges weakly to 0 in $H^{2}$ and since $T\left(z^{n}\right)=w \varphi^{n}$, we should have

$$
\int_{E}\left|w^{*}\right|^{2} d m=\int_{E}\left|w^{*}\right|^{2}\left|\varphi^{*}\right|^{2 n} d m \leq \int_{\mathbf{T}}\left|w^{*}\right|^{2}\left|\varphi^{*}\right|^{2 n} d m=\left\|T\left(z^{n}\right)\right\|^{2} \underset{n \rightarrow \infty}{\longrightarrow} 0 ;
$$

but this would imply that $w$ is null a.e. on $E$ and hence $w \equiv 0$ (see [4], Theorem 2.2), which was excluded.

Let us now prove the sufficient condition.

Assume that $m\left(\left\{\left|\varphi^{*}\right|=1\right\}\right)=0$ holds. Given $w \in H^{2}$, we can write

$$
\left\|M_{w} C_{\varphi}(f)\right\|^{2}=\int_{\mathbf{T}}\left|w^{*}\right|^{2}\left|f \circ \varphi^{*}\right|^{2} d m=\int_{\mathbf{D}}|f|^{2} d \nu
$$

where $\nu=\nu_{w}=\varphi^{*}\left(\left|w^{*}\right|^{2} m\right)$, that is $\nu(A)=\int_{\varphi^{*-1}(A)}\left|w^{*}\right|^{2} d m$. By the Carleson embedding theorem (see [2, page 129]), a necessary and sufficient condition for the operator $M_{w} C_{\varphi}: H^{2} \rightarrow H^{2}$ to be compact is that $\nu$ is a vanishing Carleson measure for $H^{2}$. We now produce a suitable $w, w \not \equiv 0$.

Let

$$
\Gamma_{h}=\{z ; 1-h \leq|z|<1\}
$$

and set

$$
F_{n}=\varphi^{*-1}\left(\Gamma_{2^{-n}}\right) \text { and } c_{n}=m\left(F_{n}\right) .
$$

Our assumption implies that $c_{n} \underset{n \rightarrow \infty}{\longrightarrow} 0$. We can hence find an increasing sequence $\left(k_{n}\right)_{n \geq 1}$ of integers such that

$$
\sum_{n=1}^{\infty} c_{k_{n}} \log n<\infty
$$

Let $\phi_{n}: \mathbf{T} \rightarrow \mathbf{R}^{+}$be defined as

$$
\phi_{n}= \begin{cases}\frac{1}{n} & \text { on } F_{k_{n}} \\ 1 & \text { on } \mathbf{T} \backslash F_{k_{n}} .\end{cases}
$$

Let $w_{n}$ be the associated outer function, satisfying $\left|w_{n}^{*}\right|=\phi_{n}$, namely $w_{n}=\exp \left(-\psi_{n}\right)$, with

$$
\psi_{n}(z)=\int_{\mathbf{T}} \frac{1+z \mathrm{e}^{-i t}}{1-z \mathrm{e}^{-i t}} \log \frac{1}{\phi_{n}(t)} d m(t)=\log n \int_{F_{k_{n}}} \frac{1+z \mathrm{e}^{-i t}}{1-z \mathrm{e}^{-i t}} d m(t) .
$$

Observe that $\mathfrak{R e} \psi_{n}(z)=\log n \int_{F_{k_{n}}} P_{z}(t) d m(t)$, where $P_{z}(t)=\frac{1-|z|^{2}}{\left|1-z \mathrm{e}^{-i t}\right|^{2}}$ is the Poisson kernel, so that $\mathfrak{R e} \psi_{n}(z) \geq 0$ and $\left|w_{n}(z)\right| \leq 1$. Moreover $\left|w_{n}^{*}\right|=\frac{1}{n}$ on $F_{k_{n}}$.

The condition (3.2) ensures that the infinite product $w=\prod_{n}^{n} w_{n}$ converges uniformly on compact subsets of $\mathbf{D}$, and defines a function $w \in H^{\infty}$, bounded by 1 and without zeros. Indeed, since $\mathfrak{R e} \psi_{n} \geq 0$, we see that

$$
\left|1-w_{n}(z)\right| \leq\left|\psi_{n}(z)\right| \leq \log n \int_{F_{k_{n}}} \frac{1+|z|}{1-|z|} d m(t)=\left(c_{k_{n}} \log n\right) \frac{1+|z|}{1-|z|}
$$


subsequently, the series $\sum\left(1-w_{n}\right)$ converges normally on compact subsets of $\mathbf{D}$, and the infinite product $\prod w_{n}$ converges uniformly on compact subsets of $\mathbf{D}$, as claimed. The weighted composition operator $M_{w} C_{\varphi}$ is bounded since $w \in H^{\infty}$.

Let finally $0<h<2^{-k_{1}}$ and $n=n(h)$ such that $2^{-k_{n+1}} \leq h<2^{-k_{n}}$. Let $\xi \in \mathbf{T}$. Then $W(\xi, h) \subseteq \Gamma_{h}$, so that

$$
\varphi^{*-1}[W(\xi, h)] \subseteq \varphi^{*-1}\left(\Gamma_{h}\right) \subseteq \varphi^{*-1}\left(\Gamma_{2^{-k_{n}}}\right)=F_{k_{n}} .
$$

As a consequence, $\left|w^{*}(u)\right| \leq\left|w_{n}^{*}(u)\right| \leq \frac{1}{n}$ for all $u \in \varphi^{*-1}[W(\xi, h)]$, and

$$
\nu[W(\xi, h)]=\int_{\varphi^{*-1}[W(\xi, h)]}\left|w^{*}\right|^{2} d m \leq \frac{1}{n^{2}} m_{\varphi}[W(\xi, h)] \leq \frac{1}{n^{2}} C h,
$$

because we know (see [2, page 129]) that $m_{\varphi}$ is a Carleson measure. This ends the proof, since $n=n(h)$ tends to $\infty$ when $h$ goes to 0 .

Remark. The previous argument can be sometimes quantified, and the degree of compactness of $M_{w} C_{\varphi}$ specified (even if there are limitations, as shown by the forthcoming Theorem 4.1).

Recall that if $T: X \rightarrow Y$ is an operator between Banach spaces $X$ and $Y$, its $n$-th approximation number $a_{n}(T)$ is defined, for $n \geq 1$, as

$$
a_{n}(T)=\inf \{\|T-R\| ; R: X \rightarrow Y \text { has rank }<n\} .
$$

When $X$ and $Y$ are Hilbert spaces, we have $a_{n}(T)=s_{n}(T)$, the $n$-th singular number of $T$.

Theorem 3.2. For each $\gamma$ with $0<\gamma<1 / 2$, there exist a non-compact composition operator $C_{\varphi}: H^{2} \rightarrow H^{2}$ and a weight $w \in H^{\infty}$ such that, for some constant $b>0$, we have

$$
a_{n}\left(M_{w} C_{\varphi}\right) \lesssim \exp \left(-b n^{\gamma}\right) .
$$

In particular, $M_{w} C_{\varphi}$ belongs to all Schatten classes $S_{p}\left(H^{2}\right), p>0$.

For the proof, we recall the following simple result.

Proposition 3.3. Let $\nu$ be a vanishing Carleson measure on D. Then

$$
a_{n}\left(J_{\nu}\right) \lesssim \inf _{0<h<1}\left(\mathrm{e}^{-n h}+\sup _{0 \leq t \leq h} \sqrt{\frac{\rho_{\nu}(t)}{t}}\right),
$$

where $J_{\nu}: H^{2} \rightarrow L^{2}(\nu)$ is the canonical inclusion. In particular, if $w \in H^{\infty}$ and $\varphi$ is a symbol, we have

$$
a_{n}\left(M_{w} C_{\varphi}\right) \lesssim \inf _{0<h<1}\left(\mathrm{e}^{-n h}+\sup _{0 \leq t \leq h} \sqrt{\frac{\rho_{\nu}(t)}{t}}\right),
$$

where $\nu=\varphi^{*}\left(\left|w^{*}\right|^{2} m\right)$ is the pull-back measure of $\left|w^{*}\right|^{2} m$ by $\varphi^{*}$.

For the proof of Proposition 3.3, we refer to [12, Theorem 5.1], where the result is given only for composition operators, but working exactly the same for inclusions, except only that we have to replace the quantity $\sqrt{\rho_{\nu}(h) / h}$ by $\sup _{0 \leq t \leq h} \sqrt{\rho_{\nu}(t) / t}$. For the special case, just use that $\left\|J_{\nu} f\right\|=\left\|\left(M_{w} C_{\varphi}\right) f\right\|$ for all $f \in H^{2}$, so there exist two contractions $U: L^{2}(\nu) \rightarrow H^{2}$ and $V: H^{2} \rightarrow H^{2}$ such that $\left(M_{w} C_{\varphi}\right)=U J_{\nu}$ and $J_{\nu}=V\left(M_{w} C_{\varphi}\right)$, and hence $a_{n}\left(M_{w} C_{\varphi}\right)=a_{n}\left(J_{\nu}\right)$. 
Proof of Theorem 3.2. We use a construction made in [8, Section 3.2]. Let $1<\beta \leq 2$ and

$$
u(t)=|\sin (t / 2)|^{\beta} .
$$

There is an analytic function $U: \mathbf{D} \rightarrow \Pi^{+}=\{\Re$ e $z>0\}$ whose boundary values are

$$
U^{*}\left(\mathrm{e}^{i t}\right)=u(t)+i \mathcal{H} u(t)
$$

where $\mathcal{H}$ is the Hilbert transform. The symbol $\varphi$ is defined, for $z \in \mathbf{D}$, as

$$
\varphi(z)=\exp (-U(z)) \text {. }
$$

By [8, Lemma 3.6 and Lemma 4.3], the composition operator $C_{\varphi}: H^{2} \rightarrow H^{2}$ is not compact.

Moreover, since $\left|\varphi^{*}\left(\mathrm{e}^{i t}\right)\right|=\exp \left(-|\sin (t / 2)|^{\beta}\right)$, we have

$$
\left|\varphi^{*}\left(\mathrm{e}^{i t}\right)\right| \geq 1-h \Longleftrightarrow|t| \leq\left(\log \frac{1}{1-h}\right)^{1 / \beta} \approx h^{1 / \beta}
$$

so, if $\Gamma_{h}$ is the annulus $\{z ; 1-h \leq|z|<1\}$, and we set

$$
F_{k}=\varphi^{*-1}\left(\Gamma_{2^{-k}}\right) \text {, }
$$

we have

$$
c_{k}:=m\left(F_{k}\right) \approx 2^{-k / \beta} .
$$

Now, let $\delta_{k}=\exp \left(-2^{k / \beta} / k^{2}\right)$. We slightly modify the example of Theorem 3.1 as follows:

$$
\phi_{k}= \begin{cases}\delta_{k} & \text { on } F_{k}, \\ 1 & \text { on } \mathbf{T} \backslash F_{k} .\end{cases}
$$

Then, the series $\sum_{k \geq 1} c_{k} \log \left(1 / \delta_{k}\right)$ converges since $c_{k} \log \left(1 / \delta_{k}\right) \lesssim 1 / k^{2}$. As in the proof of Theorem 3.1, we can define an outer function $w$ such that $\left|w^{*}\right|=\prod_{k \geq 1} \phi_{k}$. The same computation gives us, for any Carleson window $W(\xi, t)$ and for $\nu=$ $\varphi^{*}\left(\left|w^{*}\right|^{2} m\right)$ :

$$
\nu[W(\xi, t)] \lesssim \delta_{j}^{2} t, \quad \text { for } \quad 2^{-j-1} \leq t<2^{-j} .
$$

Let $0<h<1$ arbitrary. There exists an integer $l \geq 0$ such that $2^{-l-1} \leq h<2^{-l}$. Then for $0<t \leq h$, we have $2^{-j-1} \leq t<2^{-j}$ for some $j \geq l$; hence

$$
\frac{\rho_{\nu}(t)}{t} \lesssim \delta_{j}^{2} \leq \delta_{l}^{2}
$$

Therefore Proposition 3.3 gives

$$
a_{n}\left(M_{w} C_{\varphi}\right) \lesssim \inf _{l \in \mathbf{N}}\left(\mathrm{e}^{-n 2^{-l}}+\delta_{l}\right) \lesssim \inf _{l \geq 0}\left(\exp \left(-n 2^{-l}\right)+\exp \left(-2^{l / \beta} / l^{2}\right)\right) .
$$

The choice $l=\left[\frac{\beta}{(\beta+1) \log 2} \log n\right]$ gives, for some $b>0$ :

$$
a_{n}\left(M_{w} C_{\varphi}\right) \lesssim \exp \left(-b n^{1 /(\beta+1)} /(\log n)^{2}\right) .
$$

Now, if $0<\gamma<1 / 2$, we take $\beta$ such that $1<\beta<\frac{1}{\gamma}-1$ and $\beta \leq 2$. We obtain, with another $b>0$ :

as claimed.

$$
a_{n}\left(M_{w} C_{\varphi}\right) \lesssim \exp \left(-b n^{\gamma}\right)
$$

Remark 1. For $\beta<1$, since we have $m_{\varphi}\left(\Gamma_{h}\right) \approx h^{1 / \beta}$, the composition operator $C_{\varphi}$ is already compact. When $\beta=1$, we have $m_{\varphi}\left(\Gamma_{h}\right) \approx h$, but it can be checked that nevertheless $C_{\varphi}$ is compact and $\rho_{\varphi}(h)=\mathrm{O}(h / \log (1 / h))$ (see [8, Remark 3, 
page 3117]). Without doing that, we can use [8, Theorem 4.1] (which is an improvement of [9, Theorem 4.1]): there exists a compact composition operator with symbol $\widetilde{\varphi}$ such that $\left|\widetilde{\varphi}^{*}\right|=\left|\varphi^{*}\right|$; therefore $m_{\widetilde{\varphi}}\left(\Gamma_{h}\right)=m_{\varphi}\left(\Gamma_{h}\right) \approx h$.

For $\beta=1$, the above proof only gives

$$
a_{n}\left(M_{w} C_{\varphi}\right) \lesssim \exp \left(-b n^{1 / 2} /(\log n)^{2}\right)
$$

Though in this case $C_{\varphi}$ was already compact, that nevertheless allows to improve the compactness.

Remark 2. The case $\beta=2$ corresponds to the simple symbol $\varphi(z)=\frac{1+z}{2}$. Indeed, we only used in our construction the modulus of the symbol and for this $\varphi$, we have $\left|\varphi^{*}\left(\mathrm{e}^{i t}\right)\right|=|\cos (t / 2)| \approx 1-t^{2} / 8 \approx \exp \left(-|\sin (t / 2 \sqrt{2})|^{2}\right)$.

We get the following result.

Theorem 3.4. Let $\varphi(z)=\frac{1+z}{2}$. For each decreasing sequence $\left(\varepsilon_{k}\right)$ of positive numbers such that $\left(\delta_{k}\right)=\left(2^{k / 2} \varepsilon_{2^{k}}\right)$ is decreasing, there exist a weight $w \in H^{\infty}$ and a positive constant $b$ such that

$$
a_{n}\left(M_{w} C_{\varphi}\right) \lesssim \exp \left(-b n^{1 / 3} \varepsilon_{n}\right) .
$$

Proof. We only have to modify the proof of Theorem 3.2 : we replace $F_{k}$ by

$$
F_{k}=\varphi^{*-1}\left(\Gamma_{4^{k / 3}}\right)
$$

so $c_{k}=m\left(F_{k}\right) \approx 2^{-k / 3}$, and we replace $\delta_{k}=\exp \left(-2^{k / \beta} / k^{2}\right)=\exp \left(-2^{k / 2} / k^{2}\right)$ by

$$
\delta_{k}=\exp \left(-2^{k / 3} \varepsilon_{2^{k}}\right),
$$

where $\left(\varepsilon_{k}\right)_{k}$ is a given decreasing sequence of positive integers such that $\left(\delta_{k}\right)$ is decreasing. Note that, since $\left(\delta_{k}\right)$ is decreasing, we have $\varepsilon_{2^{k}} \lesssim 2^{-k / 2}$, so $\sum_{k} \varepsilon_{2^{k}}<\infty$. We get

$$
a_{n}\left(M_{w} C_{\varphi}\right) \lesssim \inf _{l \geq 0}\left(\mathrm{e}^{-n 4^{-l / 3}}+\mathrm{e}^{-2^{l / 3} \varepsilon_{2} l}\right)
$$

and, with $l=[\log n / \log 2]$, we get, since $\varepsilon_{n} \leq \varepsilon_{2^{l}}$, for some $b>0$ :

$$
a_{n}\left(M_{w} C_{\varphi}\right) \lesssim \exp \left(-b n^{1 / 3} \varepsilon_{n}\right) .
$$

For example, with $\varepsilon_{k}=1 /(\log k)^{2}$, we get $a_{n}\left(M_{w} C_{\varphi}\right) \lesssim \mathrm{e}^{\left(-b n^{1 / 3} /(\log n)^{2}\right)}$.

Theorem 3.4 improves a result of [7, Theorem 2.3], where for this symbol and a given $\alpha>0$, weights $w$ are obtained such that

$$
a_{n}\left(M_{w} C_{\varphi}\right) \lesssim\left(\frac{\log n}{n}\right)^{\alpha}
$$

\section{Hilbert-Schmidt and Schatten regularizations}

We begin with a characterization of the symbols that can give a Hilbert-Schmidt weighted composition operator.

Theorem 4.1. An analytic self-map $\varphi: \mathbf{D} \rightarrow \mathbf{D}$ can induce a Hilbert-Schmidt weighted composition operator $M_{w} C_{\varphi}$, for some weight $w \in H^{2}$, if and only if

$$
\int_{\mathbf{T}} \log \left(\frac{1}{1-\left|\varphi^{*}\right|}\right) d m<+\infty
$$


Proof. That the condition is sufficient is proved in [13, Proposition 2.5]. For sake of completeness, we recall the argument.

The hypothesis implies that there exists an outer function $w$ on $\mathbf{D}$ such that $\left|w^{*}\right|^{2}=1-\left|\varphi^{*}\right|$. Then, writing $T=M_{w} C_{\varphi}$, we have

$$
\sum_{n=0}^{\infty}\left\|T\left(z^{n}\right)\right\|^{2}=\sum_{n=0}^{\infty} \int_{\mathbf{T}}\left(1-\left|\varphi^{*}\right|\right)\left|\varphi^{*}\right|^{2 n} d m=\int_{\mathbf{T}} \frac{1}{1+\left|\varphi^{*}\right|} d m<+\infty,
$$

and $T$ is Hilbert-Schmidt, as claimed.

Let us prove the necessity of the condition. If $w \in H^{2}$ exists such that $M_{w} C_{\varphi}$ : $H^{2} \rightarrow H^{2}$ is Hilbert-Schmidt, we have in particular $\left|\varphi^{*}\right|<1 m$-almost everywhere, by the easy part of Theorem 3.1. Since $M_{w} C_{\varphi}$ is Hilbert-Schmidt, we have

$$
\sum_{n=0}^{\infty}\left\|w \varphi^{n}\right\|^{2}=\sum_{n=0}^{\infty}\left\|\left(M_{w} C_{\varphi}\right)\left(z^{n}\right)\right\|^{2}<\infty
$$

i.e.

$$
\int_{\mathbf{T}}\left|w^{*}\right|^{2} \frac{1}{1-\left|\varphi^{*}\right|^{2}} d m<\infty
$$

The following lemma, with $u=\left|w^{*}\right|^{2}, v=1-\left|\varphi^{*}\right|^{2}$ and $\alpha=1$, then shows that $\int_{\mathbf{T}} \log \frac{1}{1-\left|\varphi^{*}\right|^{2}} d m<\infty$. In fact, since $w \in H^{2}$ and $w \not \equiv 0$, Jensen's inequality tells that the first condition of that lemma is satisfied.

Lemma 4.2. Let $(\Omega, \nu)$ be a measure space and $u, v: \Omega \rightarrow(0,1]$ measurable functions such that, for some $\alpha>0$ :

$$
\int_{\Omega}|\log u| d \nu<\infty \text { and } \int_{\Omega} u v^{-\alpha} d \nu<\infty
$$

Then $\int_{\Omega}|\log v| d \nu<\infty$.

Proof. If we set $g=v^{-\alpha}$ and $f=u v^{-\alpha}$, we have

$$
0 \leq \log g=\log f+\log \frac{1}{u} \leq \log ^{+} f+|\log u| \leq f+|\log u| .
$$

By hypothesis, $f$ (which is positive) and $|\log u|$ are integrable; hence $\log g$ is integrable and

$$
\int_{\Omega}|\log v| d \nu<\infty
$$

In Theorem 4.1, we showed that for the outer function $w$ such that $\left|w^{*}\right|^{2}=1-\left|\varphi^{*}\right|$, the weighted composition operator $M_{w} C_{\varphi}$ is Hilbert-Schmidt. For this weight, we cannot expect better in general, as said by the following theorem.

Theorem 4.3. There exists a symbol $\varphi$ satisfying $\int_{\mathbf{T}} \log \left(1-\left|\varphi^{*}\right|\right) d m>-\infty$ such that, if $w$ is any outer function satisfying $\left|w^{*}\right|=1-\left|\varphi^{*}\right|$, the weighted composition operator $M_{w} C_{\varphi}$ is Hilbert-Schmidt, but $M_{w} C_{\varphi} \notin S_{p}$, for all $p<2$.

Proof. Let, for $|t| \leq \pi$ :

$$
u(t)=1-\exp \left(-\mathrm{e}^{1 /|t|}\right) .
$$

We have $0<1-\exp \left(-\mathrm{e}^{1 / \pi}\right) \leq u(t) \leq 1$; hence $\int_{-\pi}^{\pi} \log u(t) d t>-\infty$; therefore there is an outer function $\varphi \in H^{\infty}$ such that $\left|\varphi^{*}\left(\mathrm{e}^{i t}\right)\right|=u(t)$.

Moreover, we also have $\int_{\mathbf{T}} \log \left(1-\left|\varphi^{*}\right|\right) d m \approx \int_{-\pi}^{\pi} \log (1-u(t)) d t>-\infty$. Hence if $w$ is an outer function such that $\left|w^{*}\right|^{2}=1-\left|\varphi^{*}\right|$, the weighted composition operator 
$M_{w} C_{\varphi}$ is Hilbert-Schmidt. We are going to show that $M_{w} C_{\varphi}$ does not belong to any Schatten class for $p<2$.

For that, we use Theorem 2.2. The weighted composition operator $M_{w} C_{\varphi}$ can be viewed as an inclusion $J_{\nu}: H^{2} \rightarrow L^{2}(\nu)$, where $\nu=\varphi^{*}\left(\left|w^{*}\right|^{2} m\right)$. Here, we also have $d \nu(z)=(1-|z|) d m_{\varphi}(z)$.

Since $p<2$, we have

$$
\sum_{j=0}^{2^{n}-1}\left[2^{n} \nu\left(\widetilde{W}_{n, j}\right)\right]^{p / 2} \geq\left(\sum_{j=0}^{2^{n}-1} 2^{n} \nu\left(\widetilde{W}_{n, j}\right)\right)^{p / 2}=\left[2^{n} \nu\left(\widetilde{\Gamma}_{2^{-n}}\right)\right]^{p / 2},
$$

where $\widetilde{\Gamma}_{h}=\{z \in \mathbf{D} ; 1-h \leq|z| \leq 1-h / 2\}$. But $\nu\left(\widetilde{\Gamma}_{2^{-n}}\right) \approx 2^{-n} m_{\varphi}\left(\widetilde{\Gamma}_{2^{-n}}\right)$ and

$$
m_{\varphi}\left(\widetilde{\Gamma}_{h}\right) \approx \frac{1}{(\log 1 / h)(\log \log 1 / h)^{2}} .
$$

In fact, we have $\varphi^{*}\left(\mathrm{e}^{i t}\right) \in \widetilde{\Gamma}_{h}$ if and only if $h / 2 \leq \exp \left(-\mathrm{e}^{1 /|t|}\right) \leq h$, which is equivalent to

and

$$
\frac{1}{\log \log 2 / h} \leq|t| \leq \frac{1}{\log \log 1 / h}
$$

$$
\begin{aligned}
\frac{1}{\log \log 1 / h}-\frac{1}{\log \log 2 / h} & \approx \frac{1}{(\log \log 1 / h)^{2}} \log \left(1+\frac{\log 2}{\log 1 / h}\right) \\
& \approx \frac{1}{(\log 1 / h)(\log \log 1 / h)^{2}}
\end{aligned}
$$

Hence

and we obtain

$$
2^{n} \nu\left(\widetilde{\Gamma}_{2^{-n}}\right) \gtrsim \frac{1}{n(\log n)^{2}}
$$

$$
\sum_{n=0}^{+\infty} \sum_{j=0}^{2^{n}-1}\left[2^{n} \nu\left(\widetilde{W}_{n, j}\right)\right]^{p / 2} \gtrsim \sum_{n=0}^{+\infty} \frac{1}{n^{p / 2}(\log n)^{p}}=\infty
$$

since $p / 2<1$. Luecking's theorem tells that $M_{w} C_{\varphi} \notin S_{p}$.

If Theorem 4.3 does not allow to have a better behavior for $M_{w} C_{\varphi}$ than HilbertSchmidt when $w$ is an outer function such that $\left|w^{*}\right|=1-\left|\varphi^{*}\right|$, an improvement is possible by taking another weight.

Theorem 4.4. Assume that the composition operator $C_{\varphi}$ can induce a HilbertSchmidt weighted composition operator. Then there exists another weight $w \in H^{2}$ such that $M_{w} C_{\varphi} \in S_{p}$ for every $p<2$.

Proof. By Theorem 4.1, we have $\int_{\mathbf{D}} \log \frac{1}{1-|z|} d m_{\varphi}(z)<\infty$.

We will use the following version of the du Bois-Reymond lemma. For sake of completeness, we will give a short proof of this lemma, but we postpone it.

Lemma 4.5. (du Bois-Reymond lemma) Let $\mu$ be a continuous positive measure on $[0,1)$ and $f$ a positive $\mu$-integrable function. Then there exists a positive function $g$ on $[0,1)$ such that $g(t) \underset{t \rightarrow 1}{\longrightarrow} \infty$ and $\int_{0}^{1} f(t) g(t) d \mu(t)<\infty$.

So, let $g:[0,1) \rightarrow \mathbf{R}_{+}$with $g(t) \underset{t \rightarrow 1}{\longrightarrow} \infty$ and $\int_{\mathbf{D}} g(|z|) \log \frac{1}{1-|z|} d m_{\varphi}(z)<\infty$. Then there is an outer function $w$ such that $\left|w^{*}\right|=\left(1-\left|\varphi^{*}\right|\right)^{g \circ \mid \varphi^{*}}$. 
Take an integer $K>1 / p$ and let $w_{K}$ be an outer function such that $\left|w_{K}^{*}\right|=$ $\left(1-\left|\varphi^{*}\right|\right)^{K}$. We point out that

$$
\left\|w_{K}^{*}\left(\varphi^{*}\right)^{n}\right\|_{L^{\infty}(\mathbf{T})} \leq \sup _{t \in(0,1)}(1-t)^{K} t^{n} \lesssim \frac{1}{n^{K}}
$$

Hence we have, for some positive constant $C$ (depending on $K$ but not on $n$ ):

$$
\left\|\left(M_{w_{K}} C_{\varphi}\right)\left(z^{n}\right)\right\|^{2}=\int_{\mathbf{T}}\left|w_{K}^{*}\right|^{2}\left|\varphi^{*}\right|^{2 n} d m \leq \frac{C}{n^{2 K}} .
$$

It follows that $\left\|\left(M_{w_{K}} C_{\varphi}\right)\left(z^{n}\right)\right\|^{p} \leq C^{p / 2} / n^{K p}$ and hence

$$
\sum_{n=1}^{\infty}\left\|\left(M_{w_{K}} C_{\varphi}\right)\left(z^{n}\right)\right\|^{p}<\infty
$$

since $K p>1$. That implies that $M_{w_{K}} C_{\varphi} \in S_{p}$.

But $g(t) \underset{t \rightarrow 1}{\longrightarrow} \infty$, so $g(t) \geq K$ for $t$ close enough to 1 and it follows that $\left|w^{*}\right| \lesssim\left|w_{K}^{*}\right|$ (up to a constant depending on $K$ only). Hence $\left\|\left(M_{w} C_{\varphi}\right) f\right\| \lesssim\left\|\left(M_{w_{K}} C_{\varphi}\right) f\right\|$ for all $f \in H^{2}$, and it follows that $M_{w} C_{\varphi} \in S_{p}$.

Proof of Lemma 4.5. The classical du Bois-Reymond lemma states that, for any convergent series $\sum u_{n}$ of positive numbers, there is a sequence of positive numbers $\alpha_{n}$ increasing to $\infty$ such that $\sum_{n} \alpha_{n} u_{n}<\infty$. It is an easy exercise to show that. Now if $\int_{0}^{1} f d \mu<\infty$, we take $u_{n}=\int_{1-\frac{1}{n}}^{1-\frac{1}{n+1}} f d \mu$. We get the result in taking $g=$ $\sum_{n=1}^{\infty} \alpha_{n} \mathbb{1}_{\left[1-\frac{1}{n}, 1-\frac{1}{n+1}\right)}$.

Theorem 4.6. For every $p<\infty$, if $M_{w} C_{\varphi} \in S_{p}$ for some weight $w$, then there exists another weight $\widetilde{w}$ for which $M_{\widetilde{w}} C_{\varphi}$ is Hilbert-Schmidt.

Proof. For $p \leq 2$, this is obvious, with the same weight, since $S_{p} \subseteq S_{2}$. So we assume $p>2$. We have $\sum_{n=0}^{\infty}\left\|\left(M_{w} C_{\varphi}\right)\left(z^{n}\right)\right\|^{p}<\infty$, i.e.

$$
\sum_{n=0}^{\infty}\left(\int_{\mathbf{T}}\left|w^{*}\right|^{2}\left|\varphi^{*}\right|^{2 n} d m\right)^{p}<\infty
$$

When $\sum_{n=0}^{\infty}\left|c_{n}\right|^{p}<\infty$, the Hölder inequality implies that, for $\beta>1 / q$ ( $q$ is the conjugate exponent of $p$ ), we have

$$
\sum_{n=0}^{\infty} \frac{1}{n^{\beta}}\left|c_{n}\right| \leq\left(\sum_{n=0}^{\infty} \frac{1}{n^{\beta q}}\right)^{1 / q}\left(\sum_{n=0}^{\infty}\left|c_{n}\right|^{p}\right)^{1 / p}<\infty .
$$

Now,

$$
\left(1-\left|\varphi^{*}\right|^{2}\right)^{-\beta}=\sum_{n=0}^{\infty}\left(\begin{array}{c}
-\beta \\
n
\end{array}\right)(-1)^{n}\left|\varphi^{*}\right|^{2 n},
$$

and, by the Stirling formula $\left(\begin{array}{c}-\beta \\ n\end{array}\right)(-1)^{n} \approx n^{\beta-1}$. Hence if we take $\beta$ such that $1 / q<$ $\beta<1$ and set $\alpha=1-\beta$, we have $\alpha>0$ and

$$
\int_{\mathbf{T}}\left|w^{*}\right|^{2}\left(1-\left|\varphi^{*}\right|^{2}\right)^{-\alpha} d m \approx \sum_{n=0}^{\infty} \frac{1}{n^{\beta}} \int_{\mathbf{T}}\left|w^{*}\right|^{2}\left|\varphi^{*}\right|^{2 n} d m<\infty .
$$

It follows from Lemma 4.2 that $\int_{\mathbf{T}}\left|\log \left(1-\left|\varphi^{*}\right|^{2}\right)\right| d m<\infty$, and then, from Theorem 4.1, that there is a weight $\widetilde{w}$ for which $M_{\widetilde{w}} C_{\varphi}$ is Hilbert-Schmidt.

Let us put together Theorem 4.1, 4.4 and 4.6. 
Theorem 4.7. For any symbol $\varphi$, the following assertions are equivalent:

1) there is a weight $w$, with $w \in H^{2}$, such that $M_{w} C_{\varphi}$ is Hilbert-Schmidt;

2) there is a weight $\widetilde{w}$, with $\widetilde{w} \in H^{\infty}$, such that $M_{\widetilde{w}} C_{\varphi} \in S_{p}$ for all $p>0$;

3) there exist $p<\infty$ and a weight $w_{p}$, with $w_{p} \in H^{\infty}$, such that $M_{w_{p}} C_{\varphi} \in S_{p}$;

4) $\int_{\mathbf{T}} \log \frac{1}{1-\left|\varphi^{*}\right|} d m<\infty$.

As a consequence, we see that in general, the condition $m(\{|\varphi|=1\})=0$ cannot give better than a compactification.

Theorem 4.8. There exists a compactifiable symbol $\varphi$, i.e. $m\left(\left\{\left|\varphi^{*}\right|=1\right\}\right)=0$, such that, whatever the weight $w, M_{w} C_{\varphi}$ is not in any Schatten class $S_{p}$, with $p<\infty$.

Proof. It suffices to find a symbol $\varphi$ such that $m\left(\left\{\left|\varphi^{*}\right|=1\right\}\right)=0$ but such that $\int_{\mathbf{T}} \log \frac{1}{1-\left|\varphi^{*}\right|} d m=\infty$, i.e. an element of the unit ball of $H^{\infty}$ that is an extreme point of that unit ball but not an exposed point. If we set $u(t)=1-\mathrm{e}^{-1 /|t|}$ for $|t| \leq \pi$, then $0<1-\mathrm{e}^{-1 / \pi} \leq u(t) \leq 1$, so $\int_{|t| \leq \pi} \log u(t) d t>-\infty$, so there exists an outer function $\varphi \in H^{\infty}$ such that $\left|\varphi^{*}\left(\mathrm{e}^{i t}\right)\right|=u(t)$. Clearly, this function works.

\section{Weighted composition operators on $\boldsymbol{H}^{p}$}

In this section we assume that $1 \leq p<+\infty$. We are interested here in finding a characterization of the symbols that can give a weighted composition operator belonging to some specific ideal of operators. In particular, we focus on the ideal of nuclear operators and the ideal of absolutely summing operators.

First let us recall:

- An operator $T: X \rightarrow Y$ between Banach spaces $X$ and $Y$ is nuclear if there are elements $y_{n} \in Y$ and linear forms $x_{n}^{*} \in X^{*}$ with $\sum_{n=0}^{\infty}\left\|x_{n}^{*}\right\|\left\|y_{n}\right\|<\infty$ such that $T x=\sum_{n=0}^{\infty} x_{n}^{*}(x) y_{n}$ for all $x \in X$.

- An operator $T: X \rightarrow Y$ between Banach spaces $X$ and $Y$ is $r$-summing, $1 \leq r<\infty$, if there is a positive constant $C$ such that

$$
\left(\sum_{k=1}^{n}\left\|T x_{k}\right\|^{r}\right)^{1 / r} \leq C \sup _{x^{*} \in B_{X^{*}}}\left(\sum_{k=1}^{n}\left|\left\langle x^{*}, x_{k}\right\rangle\right|^{r}\right)^{1 / r}
$$

for all finite sequence $\left(x_{1}, \ldots, x_{n}\right)$ in $X$.

The main result of this section is the following theorem.

Theorem 5.1. Let $\varphi: \mathbf{D} \rightarrow \mathbf{D}$ be a symbol. The following assertions are equivalent.

(1) There exists a weight $w$ such that $M_{w} C_{\varphi}: H^{p} \rightarrow H^{p}$ is a nuclear operator for every $p \geq 1$.

(2) There exists a weight $w$ such that $M_{w} C_{\varphi}: H^{p} \rightarrow H^{p}$ is 1-summing for every $p \geq 1$ (and hence is $r$-summing for every $r \geq 1$ ).

(3) There exists a weight $w$ such that $M_{w} C_{\varphi}: H^{p} \rightarrow H^{p}$ is $r$-summing for some $r \geq 1$ and some $p \geq 1$.

(4) $\int_{\mathbf{T}} \log \frac{1}{1-\left|\varphi^{*}\right|} d m<\infty$.

Proof. Clearly (1) implies (2), which implies (3).

The weighted composition operator $\left(M_{w} C_{\varphi}\right)$ can be viewed as the Carleson embedding $J_{\nu_{p}}: H^{p} \rightarrow L^{p}\left(\nu_{p}\right)$ where $\nu_{p}=\varphi^{*}\left(\left|w^{*}\right|^{p} m\right)$ is a finite measure on $\mathbf{D}$. 
Assume (3). Then $J_{\nu_{p}}$ is actually $r$-summing on $H^{s}$ where $s=\min (2, p)$ thanks to [11, Theorem 8.4]. By [11, Proposition 2.3, 1)], we have

$$
\int_{\mathbf{T}} \frac{\left|w^{*}\right|^{p}}{\left(1-\left|\varphi^{*}\right|\right)^{s / 2}} d m=\int_{\mathbf{D}} \frac{d \nu_{p}(z)}{(1-|z|)^{s / 2}}<\infty .
$$

By Lemma 4.2, that implies that $\int_{\mathbf{T}} \log \frac{1}{1-\left|\varphi^{*}\right|} d m<\infty$ and (4) is satisfied.

Now assume that (4) is satisfied. For every $f \in H^{p}$, we denote by $\widehat{f}(n)$ its $n^{\text {th }}$ Taylor coefficient. We point out that the functional $f \in H^{p} \mapsto \widehat{f}(n)$ has norm 1 . Then, for any operator $T: H^{p} \rightarrow Y$ satisfying $\sum_{n=0}^{\infty}\left\|T e_{n}\right\|<\infty$ where $e_{n}(z)=z^{n}$, it is easy to check that $T$ is a nuclear operator.

Our assumption implies that there exists an outer function $w$ such that $\left|w^{*}\right|=$ $\left(1-\left|\varphi^{*}\right|\right)^{2}$ a.e. and we already pointed out that $\left\|w^{*}\left(\varphi^{*}\right)^{n}\right\|_{L^{\infty}(\mathbf{T})} \leq \frac{C}{n^{2}}$, for some constant $C>0$. Hence

$$
\left\|\left(M_{w} C_{\varphi}\right)\left(e_{n}\right)\right\|_{p}=\left(\int_{\mathbf{T}}\left|w^{*}\right|^{p}\left|\varphi^{*}\right|^{p n} d m\right)^{\frac{1}{p}} \leq \frac{C}{n^{2}} .
$$

We get that $\sum_{n}\left\|\left(M_{w} C_{\varphi}\right)\left(e_{n}\right)\right\|_{p}<\infty$ and hence that $\left(M_{w} C_{\varphi}\right)$ is a nuclear operator.

\section{Decompactification}

6.1. An initial example. We refer to [15, page 27] (see also [10]) for the definition of the lens map $\lambda_{\theta}$ of parameter $\theta, 0<\theta<1$.

We saw in [7, Theorem 4.1] that multiplication by a second symbol $w$ can improve the degree of compactness of a composition operator $C_{\varphi}$. For example, if $\varphi=\lambda_{\theta}$, which satisfies [10, Theorem 2.1]:

$$
\mathrm{e}^{-b_{1} \sqrt{n}} \lesssim a_{n}\left(C_{\lambda_{\theta}}\right) \lesssim \mathrm{e}^{-b_{2} \sqrt{n}}
$$

(implying in particular that $C_{\lambda_{\theta}}$ is in all Schatten classes $S_{p}\left(H^{2}\right), p>0$ ), we exhibited functions $w \in H^{\infty}$ such that

$$
\mathrm{e}^{-b_{1}^{\prime} n / \log n} \lesssim a_{n}\left(M_{w} C_{\varphi}\right) \lesssim \mathrm{e}^{-b_{2}^{\prime} n / \log n} .
$$

We wish to prove here that, conversely, multiplication by $w$ can in some sense "decompactify" $C_{\varphi}$ while keeping it bounded. We shall begin with an explicit example.

Theorem 6.1. Let $\lambda_{\theta}$ be a lens map, $0<\theta<1$, and let $w(z)=\left(1-\lambda_{\theta}(z)\right)^{a}$ where $a=\frac{1}{2}\left(1-\frac{1}{\theta}\right)<0$. Then $w \in H^{2}$ and the weighted composition operator $M_{w} C_{\lambda_{\theta}}$ is bounded but not compact on $H^{2}$, though $C_{\lambda_{\theta}}$ is in all Schatten classes $S_{p}\left(H^{2}\right), p>0$.

Proof. We first observe that $w \in H^{2}$ since $\left|1-\lambda_{\theta}^{*}(\xi)\right| \approx|1-\xi|^{\theta}$ when $\xi \in \mathbf{T}$ (see [10, Lemma 2.5]) and $2 a \theta=\theta-1>-1$. Let now $f \in H^{2}$. Then we have, formally:

$$
\left\|M_{w} C_{\varphi}(f)\right\|^{2}=\int_{\mathbf{T}}\left|1-\lambda_{\theta}^{*}(\xi)\right|^{2 a}\left|f \circ \lambda_{\theta}^{*}(\xi)\right|^{2} d m(\xi)=\int_{\mathbf{D}}|f(u)|^{2} d \mu(u),
$$

where

$$
d \mu=|1-u|^{2 a} d m_{\lambda_{\theta}}(u)
$$

with $m_{\lambda_{\theta}}=\lambda_{\theta}^{*}(m)$.

It is sufficient to prove that $\mu$ is a Carleson measure, but not a vanishing one, for $H^{2}$. We can restrict ourselves to the Carleson windows $W(1, h)$ centered at 1 . 
We know ([10, Lemma 2.5]) that, for some constants $C>c>0$, depending on $\theta$, we have $c|t|^{\theta} \leq 1-\left|\lambda_{\theta}^{*}\left(\mathrm{e}^{i t}\right)\right| \leq C|t|^{\theta}$ and $\left|\arg \left[\lambda_{\theta}^{*}\left(\mathrm{e}^{i t}\right)\right]\right| \leq C \pi|t|^{\theta}$; it follows easily that $m_{\lambda_{\theta}}[W(1, h)] \approx h^{1 / \theta}$. Hence

$$
\begin{aligned}
\mu[W(1, h)] & =\sum_{n=0}^{\infty} \mu\left[W\left(1,2^{-n} h\right) \backslash W\left(1,2^{-n-1} h\right)\right] \\
& \approx \sum_{n=0}^{\infty}\left(2^{-n} h\right)^{2 a} m_{\lambda_{\theta}}\left[W\left(1,2^{-n} h\right) \backslash W\left(1,2^{-n-1} h\right)\right] \\
& \lesssim \sum_{n=0}^{\infty}\left(2^{-n} h\right)^{2 a}\left(2^{-n} h\right)^{1 / \theta} \lesssim h \sum_{n=0}^{\infty} 2^{-n}=2 h
\end{aligned}
$$

(since $2 a+1 / \theta=1$ ), proving that $\mu$ is a Carleson measure.

On the other hand, if we consider the modified Hastings-Luecking boxes:

$$
\widetilde{\widetilde{W}}(1, h)=\{z \in \mathbf{D} ;(c / 2 C) h \leq 1-|z| \leq h \text { and }|\arg (z)| \leq \pi h\},
$$

we have $m_{\lambda_{\theta}}(\widetilde{\widetilde{W}}(1, h)) \gtrsim h^{1 / \theta}$, because if $(h / 2 C)^{1 / \theta} \leq|t| \leq(h / C)^{1 / \theta}$, we have $1-\left|\lambda_{\theta}^{*}\left(\mathrm{e}^{i t}\right)\right| \leq C|t|^{\theta} \leq h, 1-\left|\lambda_{\theta}^{*}\left(\mathrm{e}^{i t}\right)\right| \geq c|t|^{\theta} \geq(c / 2 C) h$ and $\left|\arg \left[\lambda_{\theta}^{*}\left(\mathrm{e}^{i t}\right)\right]\right| \leq$ $C \pi|t|^{\theta} \leq \pi h$, so $\lambda_{\theta}^{*}\left(\mathrm{e}^{i t}\right) \in \widetilde{\widetilde{W}}(1, h)$. It follows that

$$
\mu[W(1, h)] \geq \mu(\widetilde{\widetilde{W}}(1, h)) \gtrsim h^{2 a} m_{\lambda_{\theta}}(\widetilde{\widetilde{W}}(1, h)) \gtrsim h^{2 a} h^{1 / \theta}=h,
$$

so $\mu$ is not a vanishing Carleson measure.

6.2. The general case. We now turn to the general case, with a less explicit construction, under the following form.

Theorem 6.2. An analytic self-map $\varphi: \mathbf{D} \rightarrow \mathbf{D}$ is decompactifiable if and only if $\|\varphi\|_{\infty}=1$.

Proof. First assume that $\|\varphi\|_{\infty}<1$. Let $w \in H^{2}$ and $\left(f_{n}\right)$ a weakly null sequence in $H^{2}$; this implies that $f_{n} \underset{n \rightarrow \infty}{\longrightarrow} 0$ uniformly on compact subsets of $\mathbf{D}$, so that $\left\|f_{n} \circ \varphi\right\|_{\infty} \underset{n \rightarrow \infty}{\longrightarrow} 0$. But then

$$
\left\|M_{w} C_{\varphi}\left(f_{n}\right)\right\|_{2} \leq\|w\|_{2}\left\|f_{n} \circ \varphi\right\|_{\infty} \underset{n \rightarrow \infty}{\longrightarrow} 0 .
$$

This shows that $M_{w} C_{\varphi}$ is compact for any $w \in H^{2}$.

Now, assume that $\|\varphi\|_{\infty}=1$. We are going to show that $\varphi$ is decompactifiable. We need to find a weight $w \in H^{2}$ such that the finite (since $w \in H^{2}$ ) measure $\nu=\varphi^{*}\left(\left|w^{*}\right|^{2} m\right)$, namely:

$$
\nu(A)=\int_{\varphi^{*-1}(A)}\left|w^{*}\right|^{2} d m
$$

is Carleson (ensuring that $M_{w} C_{\varphi}: H^{2} \rightarrow H^{2}$ is bounded), but not vanishing Carleson (implying that $M_{w} C_{\varphi}: H^{2} \rightarrow H^{2}$ is not compact).

If $C_{\varphi}$ is not compact, it suffices to take $w=1$.

We now assume that $C_{\varphi}$ is compact. Then $m\left(\left\{\left|\varphi^{*}\right|=1\right\}\right)=0$. This fact and the hypothesis $\|\varphi\|_{\infty}=1$ clearly imply that $m_{\varphi}\left(\Gamma_{n}\right)>0$ for each $n$, where $\Gamma_{n}$ is the annulus $\left\{z \in \mathbf{D} ; 1-2^{-n} \leq|z|<1\right\}$. If we set

$$
C_{l}=\left\{z \in \mathbf{D} ; 1-2^{-l} \leq|z|<1-2^{-l-1}\right\},
$$


we have $\Gamma_{n}=\bigcup_{l \geq n} C_{l}$, so that $m_{\varphi}\left(C_{l}\right)>0$ for some $l \geq n$. We can therefore find an increasing sequence $\left(k_{n}\right)$ of integers such that $m_{\varphi}\left(C_{k_{n}}\right)>0$ for each $n$. Splitting in the natural way $C_{k_{n}}$ into $2^{k_{n}}$ Hastings-Luecking boxes, we can find a sequence $\left(\xi_{n}\right)$ of points of $\mathbf{T}$ such that, with $\widetilde{W}_{k_{n}}=\widetilde{W}\left(\xi_{n}, 2^{-k_{n}}\right)$ :

$$
m_{\varphi}\left(\widetilde{W}_{k_{n}}\right)>0
$$

We define our weight $w$ as an outer function $w \in H^{2}$ with boundary values $w^{*}$. Let

$$
u=1+\sum_{n=1}^{\infty} \frac{2^{-k_{n}}}{m_{\varphi}\left(\widetilde{W}_{k_{n}}\right)} \mathbb{1}_{\varphi^{-1}\left(\widetilde{W}_{k_{n}}\right)}
$$

Then $u \geq 1$, so $\log u \geq 0$, and

$$
0 \leq \int_{\mathbf{T}} \log u d m \leq \int_{\mathbf{T}}(u-1) d m=\sum_{n=1}^{\infty} 2^{-k_{n}} \leq 1<\infty ;
$$

Hence $\log u \in L^{1}(\mathbf{T})$ and there is an outer function $w \in H^{2}$ such that $\left|w^{*}\right|^{2}=u$ (see [4, page 24]).

Now, if $\nu=\varphi^{*}\left(\left|w^{*}\right|^{2} m\right)=\varphi^{*}(u m)$, we have

$$
\nu(A)=m_{\varphi}(A)+\sum_{n=1}^{\infty} \frac{2^{-k_{n}}}{m_{\varphi}\left(\widetilde{W}_{k_{n}}\right)} m_{\varphi}\left(A \cap \widetilde{W}_{k_{n}}\right),
$$

and $\nu$ is not a vanishing Carleson measure since, with $W_{k_{n}}=W\left(\xi_{n}, 2^{-k_{n}}\right)$ :

$$
\nu\left(W_{k_{n}}\right) \geq 2^{-k_{n}} \frac{m_{\varphi}\left(\widetilde{W}_{k_{n}} \cap W_{k_{n}}\right)}{m_{\varphi}\left(\widetilde{W}_{k_{n}}\right)}=2^{-k_{n}} .
$$

Let now $W=W(\xi, h)$ be an arbitrary Carleson window. Without loss of generality, we can assume $h=2^{-N}$ for some positive integer $N$, and we observe that if $z \in W \cap \widetilde{W}_{k_{n}}$, then $1-2^{-N} \leq|z| \leq 1-2^{-k_{n}-1}$, implying $k_{n} \geq N-1$. Hence $W \cap \widetilde{W}_{k_{n}}=\emptyset$ for $k_{n}<N-1$ and:

$$
\begin{aligned}
\nu(W) & =m_{\varphi}(W)+\sum_{k_{n} \geq N-1} 2^{-k_{n}} \frac{m_{\varphi}\left(\widetilde{W}_{k_{n}} \cap W\right)}{m_{\varphi}\left(\widetilde{W}_{k_{n}}\right)} \\
& \leq m_{\varphi}(W)+\sum_{k_{n} \geq N-1} 2^{-k_{n}} \leq m_{\varphi}(W)+\sum_{l \geq N-1} 2^{-l} \\
& =m_{\varphi}(W)+4 h .
\end{aligned}
$$

Since $C_{\varphi}$ is bounded, $m_{\varphi}$ is a Carleson measure and $m_{\varphi}(W)=\mathrm{O}(h)$; therefore $\nu(W)=\mathrm{O}(h)$ and hence $\nu$ is a Carleson measure. This shows that $C_{\varphi}$ is decompactified by $M_{w}$ and that completes the proof.

Remark. For $p \geq 1$, if we set $\widetilde{w}=w^{2 / p}$, then $w \in H^{p}$ and the same proof shows that the weighted composition operator $M_{\widetilde{w}} C_{\varphi}: H^{p} \rightarrow H^{p}$ is bounded but not compact.

Acknowledgement. L. Rodríguez-Piazza is partially supported by the project MTM2015-63699-P (Spanish MINECO and FEDER funds). Parts of this paper were made when he visited the Université d'Artois in Lens and the Université de Lille, in April 2018 and January 2019. It is his pleasure to thank all his colleagues in these universities for their warm welcome. 
This work is also partially supported by the grant ANR-17-CE40-0021 of the French National Research Agency ANR (project Front).

\section{References}

[1] Amar, É, and A. Lederer: Points exposés de la boule unité de $H^{\infty}(D)$. - C. R. Acad. Sci. Paris Sér. A-B 272, 1971, A 1449-A 1452.

[2] Cowen, C., and B. MacCluer: Composition operators on spaces of analytic functions. Studies in Advanced Mathematics, CRC Press, 1994.

[3] Diestel, J., H. Jarchow, and A. Tonge: Absolutely summing operators. - Cambridge Stud. Adv. Math. 43, Cambridge Univ. Press, Cambridge, 1995.

[4] Duren, P. L.: Theory of $H^{p}$ spaces. - Dover Publ. Inc., Mineola-New York, 2000.

[5] Gallardo-Gutiérrez, E. A., R. Kumar, and J. R. Partington: Boundedness, compactness and Schatten-class membership of weighted composition operators. - Integral Equations Operator Theory 67:4, 2010, 467-479.

[6] Hu, B., L. H. Khoi, and K. Zhu: Frames and operators in Schatten classes. - Houston J. Math. 41:4, 2015, 1191-1219.

[7] Lechner, G., D. Li, H. Queffélec, and L. Rodríguez-Piazza: Approximation numbers of weighted composition operators. - J. Funct. Anal. 274:7, 2018, 1928-1958.

[8] Lefèvre, P., D. Li, H. Queffélec, and L. Rodríguez-Piazza: Some examples of compact composition operators on $H^{2}$. - J. Funct. Anal. 255:11, 2008, 3098-3124.

[9] Lefèvre, P., D. Li, H. Queffélec, and L. Rodríguez-Piazza: Composition operators on Hardy-Orlicz spaces. - Mem. Amer. Math. Soc. 207:974, 2010.

[10] Lefèvre, P., D. Li, H. Queffélec, L. Rodríguez-Piazza: Some new properties of composition operators associated with lens maps. - Israel J. Math. 195:2, 2013, 801-824.

[11] Lefèvre, P., and L. Rodríguez-PiazzA: Absolutely summing Carleson embeddings on Hardy spaces. - Adv. Math. 340, 2018, 528-587.

[12] Li, D., H. QueffÉlec, and L. Rodríguez-Piazza: On approximation numbers of composition operators. - J. Approx. Theory 164:4, 2012, 431-459.

[13] Li, D., H. Queffélec, and L. Rodríguez-Piazza: Some examples of composition operators and their approximation numbers on the Hardy space of the bidisk. - Trans. Amer. Math. Soc. $372: 4,2019,2631-2658$.

[14] Luecking, D. H.: Trace ideal criteria for Toeplitz operators. - J. Funct. Anal. 73:2, 1987, $345-368$

[15] Shapiro, J.: Composition operators and classical function theory. - Universitext, Tracts in Mathematics, Springer-Verlag, 1993.

Received 12 April 2019 • Accepted 22 January 2020 